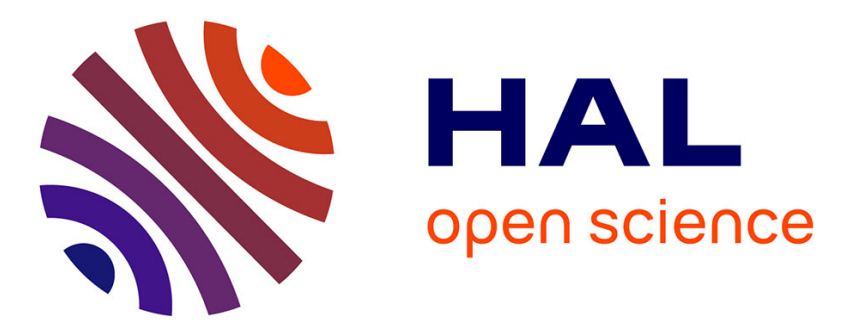

\title{
Constraining upper-limb synergies of hemiparetic patients using a robotic exoskeleton in the perspective of neuro-rehabilitation
}

\author{
Vincent Crocher, Anis Sahbani, J. Robertson, Agnès Roby-Brami, Guillaume
} Morel

\section{To cite this version:}

Vincent Crocher, Anis Sahbani, J. Robertson, Agnès Roby-Brami, Guillaume Morel. Constraining upper-limb synergies of hemiparetic patients using a robotic exoskeleton in the perspective of neurorehabilitation. IEEE Transactions on Neural Systems and Rehabilitation Engineering, 2012, 20 (3), pp.247-257. 10.1109/TNSRE.2012.2190522 . hal-00731067

\section{HAL Id: hal-00731067 https://hal.science/hal-00731067}

Submitted on 11 Sep 2012

HAL is a multi-disciplinary open access archive for the deposit and dissemination of scientific research documents, whether they are published or not. The documents may come from teaching and research institutions in France or abroad, or from public or private research centers.
L'archive ouverte pluridisciplinaire HAL, est destinée au dépôt et à la diffusion de documents scientifiques de niveau recherche, publiés ou non, émanant des établissements d'enseignement et de recherche français ou étrangers, des laboratoires publics ou privés. 


\title{
Constraining upper limb synergies of hemiparetic patients using a robotic exoskeleton in the perspective of neuro-rehabilitation
}

\author{
V. Crocher, A. Sahbani, J. Robertson, A. Roby-Brami and G. Morel
}

\begin{abstract}
The aim of this article was to explore how an upper limb exoskeleton can be programmed to impose specific joint coordination patterns during rehabilitation. Based on rationale which emphasizes the importance of the quality of movement coordination in the motor relearning process, a robot controller was developed with the aim of reproducing the individual corrections imposed by a physical therapist on a hemiparetic patient during pointing movements.

The approach exploits a description of the joint synergies using Principal Component Analysis (PCA) on joint velocities. This mathematical tool is used both to characterize the patient's movements, with or without the assistance of a physical therapist, and to program the exoskeleton during active-assisted exercises. An original feature of this controller is that the hand trajectory is not imposed on the patient: only the coordination law is modified. Experiments with hemiparetic patients using this new activeassisted mode were conducted. Obtained results demonstrate that the desired inter-joint coordination was successfully enforced, without significantly modifying the trajectory of the end point.
\end{abstract}

\section{INTRODUCTION}

In France, each year 120000 to 150000 persons suffer a brain lesion of vascular origin [1]. About half of these patients are left with a disability of the upper limb [2], [3]. Recent advances in the knowledge of activity-dependent neural plasticity have led to the development of new neurorehabilitation techniques. In this area, technology-assisted therapies, namely virtual reality [4], Functional Electrical Stimulation (FES) [5] and robotics [6], [7], [8], are being intensively explored. Robotic devices offer interesting perspectives in the field of neurorehabilitation by complementing and possibly augmenting the effectiveness of manual physical therapy because they afford many more movement repetitions [9], [10], [11], [12]. Recent extensive clinical testing of the InMotion robot has shown significant improvements in arm motor capacity after a robot therapy but the qualitative benefit of robotics over manual therapy has not been proved [13], [14].

These studies have identified a crucial feature of robotic devices: the ability of fine interaction with the patient. They have been carried out using 2D manipulanda connected to the patient's hand, whereas conventional physical therapy for the upper limb involves interactions with the impaired limb not only at the level of the hand but also, distributively, along the

V. Crocher, A. Sahbani and G. Morel are with Institute of Intelligent Systems and Robotics (CNRS - UMR 7222), University Pierre \& Marie Curie, Paris, France crocher, sahbani, moreldisir.fr

J. Robertson and A. Roby-Brami are with Laboratory of Neurophysics and Physiology (CNRS - UMR 8119), University Paris Descartes, Paris, France and with Raymond Poincare Hospital, Garches, France johanna.robertsonerpc.aphp.fr agnes.roby-brami@univ-paris5. fr

A. Roby-Brami is supported by INSERM.

The support of the French National Agency for research, ANR, program PSIROB-ROBO-0003, to the Brahma Project is gratefully acknowledged. upper arm and lower arm. For this reason, robotic exoskeletons are of particular interest, since they offer the opportunity to distribute interaction along the assisted limb. It is expected that exoskeletons will provide the means to help patients to recover not only end point (hand) control, but also interjoint synchronization which is disrupted in these patients [15]. Correcting upper limb joint synergies is essential in order to reduce abnormal inter-joint coupling between the shoulder and elbow and to retrain flexible, task dependent synergies [16].

Research on the development of exoskeletons for rehabilitaiton which can interact at the joint level has so far mainly focused on design [17], [18], [19], [20]. In this paper we shall use the ABLE exoskeleton [21], a cable actuated 4 Degrees of Freedom (DoF) robot exhibiting high backdrivability.

Literature regarding the way an exoskeleton can be programmed and controlled in order to properly interact with patients is lacking. In fact, the state-of-the art is limited to joint impedance control and optimal joint trajectory generation [22], [23]. All the controllers previously mentioned have the drawback of requiring the definition, prior to any movement of either a desired trajectory or desired interaction forces [24]. This contradicts the need to react interactively to a voluntary movement produced by the patient. Most importantly, to the authors' knowledge, the question of inter-joint coordination has never been explicitly accounted for in the design of an interactive controller for exoskeleton devices.

The central question of the present paper is how can an exoskeleton deal with the problem of inter-joint coordination. This question stems from physiopathological observations and clinical requirements for neurorehabilitation. We propose here a controller based on velocity synergies designed to modify the coordination of active movements of the user. The effect of this controller was previously studied in healthy subjects [25], and its capacity to change joint coordination with little effect on hand kinematics during pointing movements was demonstrated. This paper shows the experimental application of this concept in hemiparetic patients in the context of motor rehabilitation.

\section{STROKE AND INTERJOINT COORDINATION}

\section{A. Motor consequences of brain lesions}

When the lesion due to stroke includes the motor areas of the brain it induces a motor impairment of the body on the opposite side of the brain lesion (hemiplegia). During the weeks following the lesion, the symptoms usually recover spontaneously but partially and inconstantly (hemiparesis). Many patients are left with a disability of the upper limb which affects global movements (e.g. reaching) and hand dexterity. Negative symptoms (weakness, loss of individual joint control) 
are accompanied by positive symptoms with excessive muscular contractions: spasticity (a velocity-dependent increase of stretch-reflex) [26] and abnormal synergies [27]. Recent quantitative analyses of movement showed decreased velocity of the hand with excessive segmentation and decreased smoothness [28]. This observation suggests that the motor impairment after stroke is due to the absence of temporal blending of sub-movements [28]. Another hypothesis is that the temporal disorganization of the movements is a consequence of the disruption of the spatial inter-joint coordination particularly the coordination between elbow extension and shoulder flexion (Fig. 1) [29], [30], [31].
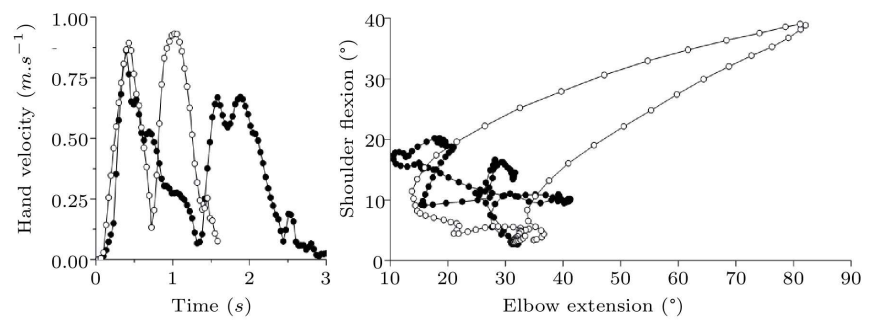

Fig. 1. Examples of movement kinematics in a healthy subject (white circles) and a hemiparetic patient (black circles). The picture displays a reaching movement and return. Left: velocity profile of the hand trajectory, Right: relationship between elbow extension and shoulder flexion.

\section{B. Normal or abnormal synergies?}

On the one hand, hemiparetic patients frequently have abnormally fixed synergies. The classical clinical descriptions distinguish between the flexor synergy (coupling of elbow flexion with shoulder abduction-extension-external rotation) and the extensor synergy (coupling of elbow extension with shoulder adduction-flexion-internal rotation) that can be triggered by any effort to move [27], [32]. During attempts to make forward reaching movements, the flexion synergy may impede elbow extension and induce excessive arm abduction bringing the elbow to an excessive height [33]. Recent studies have shown that these pathological synergies could be due to an abnormal coupling of reflexes between elbow and shoulder muscles, enhanced by activity [34], [35], [36].

On the other hand, the normally flexible interjoint coordination is disrupted in hemiparetic patients [15], [29], [30]. The building of synergies is a fundamental aspect of human motor control to overcome the redundancy of the motor system, as pointed originally by Bernstein [37]. A synergy can be expressed at several spaces: muscular activity, joint positions, joint velocities or joint torques. A recent view attributes three essential properties to the concept of synergy: the sharing pattern of rotations; flexibility allowing automatic compensation between elements and task dependency [38]. The sharing property can be represented using principal component analysis (PCA): given the space $\mathbf{E}$ of redundant kinematic variables coding a given human motion, only a small subspace of $\mathbf{E}$ is explored during natural movements. This subspace, the dimension of which corresponds to the number of DoFs required for the task, is spanned by a few orthogonal vectors (the Principal Components (PCs)). PCA for synergy description is often operated in the joint position space. For example, in [39], among the ten DoF coding the complex joint motion of the human arm, it is evidenced that 3 principal components explain $85 \%$ of the posture during a catching task. The flexibility property of the synergies accounts for the automatic error compensation or motor equivalence ensuring stable performance variables from trial-to-trial variability. This can be evidenced by the uncontrolled manifold (UCM) method which analyses the structure of variability and reveals synergies characterized by stability against perturbations and flexibility to solve concurrent tasks [38], [40], [41]. Reisman et al. recently performed two studies of synergies in hemiparetic patients using the PCA and UCM tools. They observed changed kinematic patterns of joint coupling [42] and a deficit in the error compensation properties that appeared only with increased constraints depending on the workspace location [43].

\section{Are stroke patients able to re-learn appropriate synergies?}

The ability of stroke patients to re-learn a specific motor pattern has long being disputed. The initial recovery was commonly attributed to "spontaneous" biological factors. During the last decade, the demonstration of activity-dependent cerebral plasticity and the recognition of an aptitude for recovery in hemiparetic patients have led to the proposition of many new rehabilitation methods based on motor learning theories (review in Huang and Krakaeur [44]). These methods are based on well-known paradigms of learning in healthy subjects, particularly the adaptation of reaching in force fields generated by a planar robotic manipulandum. Accordingly, most studies of robot-mediated learning of upper limb gestures in hemiparetic patients have been performed and analyzed at the level of the end point trajectory.

The recent advances in motor learning and plasticity also question the practice of manual physical therapy. The recent Motor Relearning Approach [44] differs from classical Bobath therapy [45]: it focuses more on goal directed movements, (i.e. the movement of the end point is controlled in the task space) while the Bobath approach insists particularly on the quality of the coordination. The facilitation techniques used in the Bobath approach are based on the handling skills of the physical therapist while the patient is more regarded as active learner in the Motor Relearning Approach. However, despite these different theoretical backgrounds, the practical content of each concept has a lot in common [46]. In particular, the physical therapist supports the weight of the upper limb by simultaneously holding the upper and the lower arm in order to mobilize the upper arm or to assist voluntary reaching movements. In addition, this supporting gesture is important for the prevention of algoneurodystrophy due to shoulder subluxation. The compared effectiveness of Bobath and Motor Relearning approaches is still disputed [47], [48]. A combination of these approaches would imply insisting on the quality of coordination, monitored or guided by a therapist, while the patient practices a motor learning program [49].

Although there is some evidence that robotic therapy should be effective for the recovery of the shoulder-elbow syn- 
ergy [16], the potential for and conditions of the reacquisition of appropriate and flexible interjoint synergies in hemiparetic patients remains an open question. Some clinical experiments with trunk restraint suggest that hemiparetic patients might recover a shoulder-elbow synergy and the amount of elbow extension after training [50], [51]. Training associated with functional electrical stimulation also promotes the recovery of shoulder-elbow coordination [52]. These elements confirm the importance of neurorehabilitation at the inter-joint level. Further methodological developments are needed to analyze and to promote the quality of movement coordination during therapy, whether manual or assisted. An interesting proposition comes from the work of Culmer et al. [24] who quantified the kinematics and kinetics of the supportive action of a therapist during passive assisted movements. This work gave interesting ranges of values for the torques and angular excursion implied during passive mobilization.

\section{Methods FOR MODELING AND CONTROL}

In this section, the exoskeleton controller is described. It is based on a mathematical representation of synergies. Since the objective is to provide corrections for movements without any reference to a predefined trajectory, the synergies are described in the joint velocity space rather than in the joint position space. Without losing generalisabilty, the case of 3 DoFs pointing tasks is considered in the next.

\section{A. Characterizing synergies}

1) Analysis of inter-joint coordinations using PCA on joint velocities: A pointing task in $3 \mathrm{D}$, when the hand orientation is not constrained, requires 3 DoFs. Considering an $n$ joint exoskeleton with $n>3$, redundancy occurs with respect to the task. In order to capture the way the redundancy is solved, PCA can be performed on the robot joint velocity vector $\dot{\mathbf{q}}_{r}(t)$, as detailed in [25]: during several pointing movements, the robot joint velocities are recorded and a PCA is carried out in order to identify an orthogonal basis in the joint space, composed of $n$ principal components (PCs) $\mathbf{p}_{i}$. The $n \mathbf{p}_{i}$ vectors are ordered in such a way that the direction $\mathbf{p}_{1}$ represents the most variance of the recorded data whereas the direction $\mathbf{p}_{n}$ represents the least variance. For a given movement, the following decomposition holds [39]:

$$
\dot{\mathbf{q}}_{r}(t)=\sum_{i=1}^{n} w_{i}(t) \mathbf{p}_{i} \approx \sum_{i=1}^{d} w_{i}(t) \mathbf{p}_{i}
$$

where $d<n$. Experiments conducted with healthy subjects [25] showed that only $d=3$ PCs are necessary in order to describe a class of $3 \mathrm{D}$ pointing movements similar to those considered in the present paper, without significantly losing information.

An alternative formulation for the use of PCs consists of defining a matrix $\mathbf{C} \in \mathbb{R}^{(n-3) \times n}$ by:

$$
\mathbf{C}=\left[\begin{array}{l}
\mathbf{p}_{4}^{T} \\
\vdots \\
\mathbf{p}_{n}^{T}
\end{array}\right] \text {. }
$$

This matrix $\mathbf{C}$ is a base of the (mostly) unused subspace of the pointing task. It is then interesting to notice that Eq. (1) is equivalent to:

$$
\mathbf{C} \dot{\mathbf{q}}_{r}(t) \approx \mathbf{0}
$$

where $\mathbf{C}$ clearly appears to be a constraint matrix for the joint velocities. For each subject performing a series of pointing movements with an exoskeleton, a constraint matrix $\mathbf{C}$ can be identified using PCA. When the exoskeleton is programmed to be transparent, namely to not alter the subject's intended movements, [53], the corresponding matrix is called the natural constraint and noted $\mathbf{C}_{n}$. Note that in the next, we consider, without losing generalisabilty, a 4-DoF exoskeleton. In this case, matrix $\mathbf{C}$ is reduced to a line vector:

$$
\mathbf{C}=\mathbf{p}_{4}^{T}
$$

2) Metrics: In order to evaluate whether the two methods of coordinating movement are similar, a measure was developed to quantify the "distance" between $\mathbf{C}_{1}$ and $\mathbf{C}_{2}$. The distance $\psi(\mathbf{U}, \mathbf{V})$ between two subspaces of a vector space spanned by their orthonormal bases $\mathbf{U}$ and $\mathbf{V}$ can be defined as follows [39]:

$$
\psi(\mathbf{U}, \mathbf{V})=\sqrt{1-S_{\min }^{2}\left(\mathbf{U}^{\mathbf{T}} \mathbf{V}\right)},
$$

where $S_{\text {min }}$ is the minimal singular value of the matrix $\mathbf{U}^{\mathbf{T}} \mathbf{V}$. This function represents the sine of the minimal angle between $\mathbf{U}$ and V. It is zero if the two subspaces are equal; increasing values of $\psi$ indicates an increasing difference between the two subspaces, while $\psi=1$ denotes that they are orthogonal. It is important to note that in the case of two subspaces respectively spanned by $\mathbf{U}$ and $\mathbf{V}$ :

$$
\psi(\mathbf{U}, \mathbf{V})=\psi(\mathbf{P}, \mathbf{Q})
$$

where $\mathbf{P}$ (resp. Q) is the orthogonal complement of $\mathbf{U}$ (resp. V). Applying this property to two vector spaces $\mathbf{p}^{1}$ and $\mathbf{p}^{2}$ obtained by PCA, this yields:

$$
\psi\left(\mathbf{p}_{1: 3}^{1}, \mathbf{p}_{1: 3}^{2}\right)=\psi\left(\mathbf{p}_{4}^{1}, \mathbf{p}_{4}^{2}\right)=\psi\left(\mathbf{C}_{1}^{T}, \mathbf{C}_{2}^{T}\right) .
$$

Throughout the paper, $\psi\left(\mathbf{C}_{1}^{T}, \mathbf{C}_{2}^{T}\right)$ will be used as a measure of the difference between the two constraints characterized by $\mathbf{C}_{1}$ and $\mathbf{C}_{2}$ respectively.

We felt that it was pertinent to quantify the distance between the two constraints with reference to a third one, noted as $\mathbf{C}_{\text {ref }}$. If the subject's natural constraint is $\mathbf{C}_{n}$, and the exoskeleton is programmed to impose a constraint $\mathbf{C}_{\text {ref }}$ (see how in Sec. III-B), the velocities recorded during experiments can be used to identify the resulting matrix $\mathbf{C}_{1}$. The ratio:

$$
r=\frac{\psi\left(\mathbf{C}_{1}^{T}, \mathbf{C}_{\mathrm{ref}}^{T}\right)}{\psi\left(\mathbf{C}_{n}^{T}, \mathbf{C}_{\mathrm{ref}}^{T}\right)}
$$

will be used in order to indicate whether the subject respected the applied constraint, while taking into account the amount of the change imposed by the robot with respect to the subject's natural movement. 


\section{B. Robot control}

In this section, we describe a robot controller capable of imposing a given constraint $\mathbf{C}$, defined by Eq. (2) by applying dissipative joint constraints

1) Low level torque control: The proposed control law was developed in the joint space. It is assumed that the device is torque-controllable i.e. the control input to the system is defined by the vector $\tau_{m} \in \mathbb{R}^{n}$ regrouping $n$ joint motor torques $\tau_{m_{i}}, i \in\{1 \ldots n\}$. This is required to obtain proper backdrivability.

In order to be able to apply a torque command on exoskeleton joints, it is first essential to eliminate perturbations due to the robot dynamics. Indeed the robot arm has its own weight and its actuators exhibit friction. At the lowest level, the control law is composed of three different terms:

- A gravity compensation torque $\tau_{g}$, calculated from joint positions and robot element masses;

- A friction compensation torque $\tau_{f}$, calculated from joint speeds and actuator viscosity identification;

- A commanded torque $\tau_{c}$.

The motor torque $\tau_{m}$ is the sum of these three torques:

$$
\tau_{m}=\tau_{g}+\tau_{f}+\tau_{c}
$$

as shown on Fig. 2.

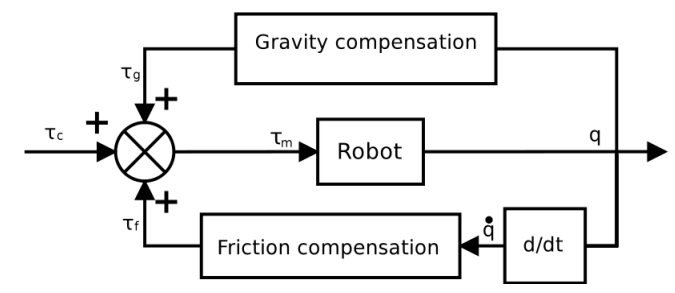

Fig. 2. The control loop with the three different torques.

2) Explicit integration of kinematic constraints into viscous fields: Only a short description of the controller is given here, details can be found in [54]. A joint viscous field is used to react to joint velocities:

$$
\tau_{c}=-\mathbf{K} \dot{\mathbf{q}}_{r}
$$

where $\mathbf{K} \in \mathbb{R}^{n \times n}$ is a viscosity matrix. In the next, we show how to compute this control law in order to impose a given arbitrary constraint $\mathbf{C} \in \mathbb{R}^{l \times n}$.

Assuming that the exoskeleton is moved by the subject, at a given time, the joint velocity is $\dot{\mathbf{q}}_{r}$. If $\mathbf{C} \dot{\mathbf{q}}_{r}=\mathbf{0}$, then the resistive torque shall be null, because the subject's motion satisfies the constraint. In any other case, a corrective torque will be applied to correct the velocity $\dot{\mathbf{q}}_{r}$. The smallest velocity correction $\dot{\mathbf{q}}_{c}$ that brings $\dot{\mathbf{q}}_{r}$ back to the constraint is obtained from the orthogonal projection of $\dot{\mathbf{q}}_{r}$ onto the orthogonal direction of the constraint $\mathbf{C}$ :

$$
\dot{\mathbf{q}}_{c}=\mathbf{C}^{+} \mathbf{C} \dot{\mathbf{q}}_{r}
$$

where $\mathbf{C}^{+}$is the $\mathbf{C} \in \mathbb{R}^{l \times n}$ matrix pseudo-inverse given by:

$$
\mathbf{C}^{+}=\mathbf{C}^{T}\left(\mathbf{C C}^{T}\right)^{-1}
$$

The torque is thus chosen to be proportional to $\dot{\mathbf{q}}_{c}$, namely:

$$
\tau_{c}=-k \mathbf{C}^{+} \mathbf{C} \dot{\mathbf{q}}_{r}
$$

where $k$ is a scalar viscosity coefficient tuning the intensity of the assistance. Note that when $l=n-1$, which corresponds to the experimental situation in the present paper, the control law is simplified to:

$$
\tau_{c}=-k \mathbf{C}^{T} \mathbf{C} \dot{\mathbf{q}}_{r}
$$

\section{EXPERIMENTAL METHODS}

\section{A. Patients}

Seven patients with right-sided hemiparesis were included in this study ( 2 women, 5 men, aged 42-70), all previously right handed. The time elapsed after brain injury of vascular or traumatic origin ranged from 5 to 100 months. Clinical and functional data are summarized in Table I. Briefly, one patient $(\sharp 2)$ had a severe hemiplegia and was not able to produce any voluntary movements. Five patients $(\sharp 3$ to 7 ) had a moderate to severe hemiparesis, with various associated motor disorders, but retained the ability to make at least gross reaching movements. Finally, one patient $(\sharp 1)$ had a mild hemiparesis with a slight limitation of voluntary movement and no associated disorders. We chose participants with a wide range of impairments in order to test the system in different clinical conditions. The most severely affected patient $(\sharp 2)$ was not able to complete the active part of the protocol: he carried out only steps 3 and 6 (see below). Therefore, his data were not included in the statistical analysis.

The study was approved by the local ethics committee and the French agency for Health products (AFSSAPS). All patients provided informed consent before participating in the study.

\section{B. Experimental apparatus : $A B L E$}

ABLE is a 4-DoF exoskeleton developed by CEA-LIST, described in details in [21]. All four DoF are actuated by a motor and a screw-cable mechanical transmission. Optical incremental encoders mounted on each joint enable the calculation of joint positions and speeds by derivation. The first three axes are coinciding at the robot shoulder center and the fourth axis, parallel to the third one, is located at the elbow level (see Fig. 3).

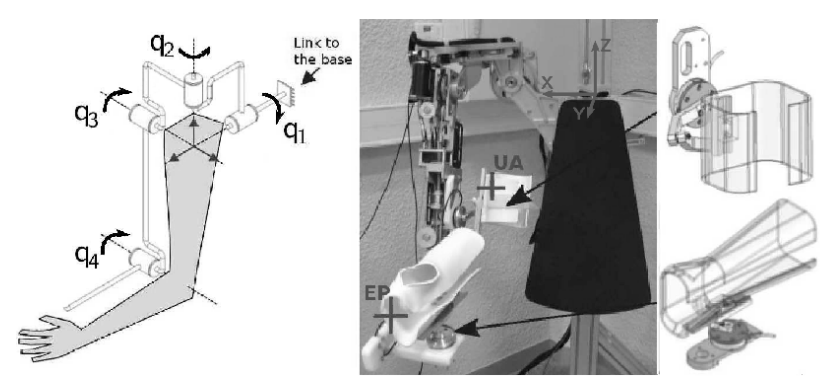

Fig. 3. ABLE kinematics (left), photography with the two splints, the two passive mechanisms and the associated frames (center) and details of the two passive mechanisms with splints (right). 
TABLE I

CLINICAL CHARACTERISTICS OF PATIENTS

\begin{tabular}{|c|c|c|c|c|c|c|c|c|c|}
\hline $\mathbf{N}$ & Gender-Age & Cause & Time & Passive limitation & MAS & Sens & MRC & FAT & $\overline{\mathbf{B I}}$ \\
\hline 2 & M-64 & Ischemia & 7 & $\bmod$ & 2 & $\bmod$ & 0 & 0 & 95 \\
\hline 7 & F-42 & Ischemia & 100 & no & 2 & no & 2 to 4 & 1 & 95 \\
\hline 5 & M-53 & Ischemia & 16 & $\bmod$ & 2 & $\bmod$ & 2 to 3 & 1 & 95 \\
\hline 6 & M-44 & Brain injury & 75 & $\bmod$ & 4 & severe & 1 to 4 & 3 & 95 \\
\hline 4 & M-70 & Ischemia & 5 & $\bmod$ & 1 & no & 3 to 4 & 3 & 100 \\
\hline 3 & M-66 & Haematoma & 12 & $\bmod$ & 2 & $\bmod$ & 3 to 4 & 4 & 90 \\
\hline 1 & F-69 & Intracranial hemorrhage & 6 & no & 0 & no & 4 to 5 & 5 & 100 \\
\hline
\end{tabular}

The table indicates the gender and age of the patients, the cause of the brain lesion, the time elapsed from the injury (in months), the presence of limited range of passive motion (mod: moderate), the spasticity measured by the Modified Ashworth Scale (MAS - from 0: no spasticity to 4: severe spasticity), the occurrence of somatosensory deficit (sens), the evaluation of the active force of the main upper limb muscle groups (MRC score, from 0 : paralysis to 5 : normal force), the Frenchay Arm Test (FAT) results that evaluate the capacity to carry out 5 elementary gestures (0-5), the Barthel Index (BI) that evaluates functional independence (0-100). Patients are ranked according to increasing function as evaluated by the FAT whereas the first column, $\mathrm{N}$ indicates the recruitment order

In order to use the exoskeleton, the subjects wears two splints which are attached to the device. One splint is worn at the level of the biceps and the other is worn on the wrist, in order to lock the wrist motion, as shown in Fig. 3. The splints are connected to the exoskeleton via two fixations. Each fixation consists of 4 passive DoFs made of a ball joint followed by a prismatic joint. These passive mechanisms, avoid hyperstaticity between the robot structure and the human limb, as demonstrated in [55]. Moreover, previous experiments with healthy subjects [53] demonstrated that the natural movements of subjects are only moderately affected a null torque $\tau_{c}$ is applied to the exoskeleton. This is due to the high backdrivability of the power transmission, the removal of hyperstatic forces by the fixation mechanisms and the gravity compensation presented in Sec. III-B.

A real time controller, implemented on a PC104 computer with two endowed 3-axis torque control cards, under RTLinux operating system was used to run the robot control loop at $1 \mathrm{kHz}$ while data (joint positions, speeds and torques) were recorded for post treatment at $500 \mathrm{~Hz}$.

\section{Set-up}

During the experiments, patients sat on a stool with their backs supported by a large board on which the exoskeleton base body is mounted. The exoskeleton shoulder (first three axes) was positioned at the level of the patient's right shoulder. The patient was then equipped with the two splints.
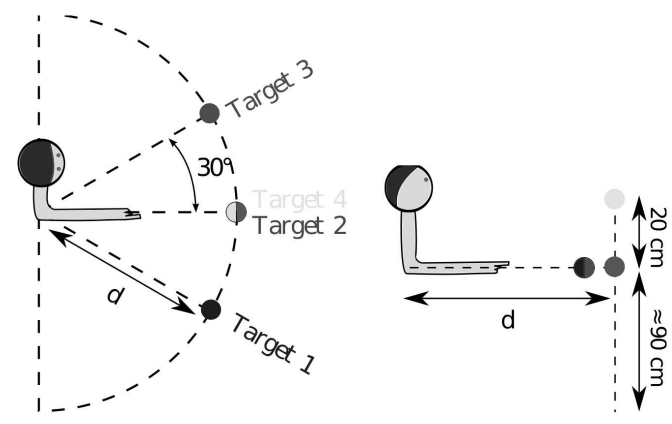

Fig. 4. Experimental setup, upper view and side view, with the 4 targets and the subject in the starting configuration. $d$ represents the adjustable distance to the targets.
The experiments were performed during one session which was divided into 6 steps. For each step the patient was asked to make pointing movements toward one of 4 different targets materialized by $2 \mathrm{~cm}$ plastic marks fixed on a movable vertical rod and positioned in the patient's workspace. The first target was placed $30^{\circ}$ to the left of the sagittal plane (internal target), the 2nd and 4th in the sagittal plane (central and high targets) and the $3 \mathrm{rd}$ target $30^{\circ}$ to the right (external target). The first three targets were placed approximately at elbow height at starting posture, and the 4 th was $20 \mathrm{~cm}$ above target 2 . The distances of the rods were adjusted for each patient so that he/she could reach it without pain, when his/her movement was guided by the therapist. Fig. 4 presents a schematic view of the setup.

The starting position was with the forearm placed laterally on an adjustable support at the level of the wrist, and approximately parallel to the thigh. The instruction was to point grossly with the base of the flexed fingers and to make three successive reaching movements between the initial hand position and the target. If the patient was unable to touch the target, he/she was asked to reach as close as possible to it. The patients were allowed to rest briefly between trials if necessary.

\section{Measurement protocol}

The experimental protocol was composed of six steps (corresponding to the different modes of control) during which the patient performed pointing movements toward the four targets in the same order (1 to 4$)$.

The aim of the first part of the protocol was to calculate interjoint coordination in hemiparetic patients.

1) The first mode was a condition, without the robot, in order to record the spontaneous movements of the patient. The patients were wearing the splints but were not connected to the robot.

2) In the 2nd mode, the splints were connected to the robot via the fixations and the exoskeleton was controlled in the so-called transparent mode. Namely, the control law (9) was applied with gravity compensation and no active torque $\left(\tau_{c}=\right.$ $0)$. The robot data recorded during this mode were used to compute the patient's natural constraint, $\mathbf{C}_{n}$, using PCA, as described in part III-A. 
3) In the 3rd mode, the patient, still connected to the robot, was instructed to remain passive while the therapist held his/her arm and performed the task by guiding the patient's arm. During this step, the robot was still in the transparent mode, characterized by a minimal resistance to movements. The robot data recorded during this mode were used to compute a new constraint called "therapeutic constraint" and noted $\mathbf{C}_{t}$.

The second part of the protocol evaluated the ability of the proposed controller (Sec. III-B) to correct the upper limb synergies of the patients. This part was performed on the same day, immediately after the fifteen minutes needed for the data transfer and the computation of $\mathbf{C}_{n}$ and $\mathbf{C}_{t}$. During the following experiments the patient was not informed of the strategy applied by the robot.

4) In the 4th mode, the patient carried out the task while wearing the exoskeleton. The control law was activated to apply the natural constraint $\mathbf{C}_{n}$, computed from the data recorded in mode 2 (with $k=1.0$ ).

5) In the 5th mode, the patient also carried out the task with the exoskeleton. The control law was activated to apply the "therapeutic constraint" $\mathbf{C}_{t}$ computed from the data recorded in mode 3 (with $k=1.0$ ).

6) In the 6th mode, the movement was imposed by the robot which simply replayed the trajectories recorded by the exoskeleton in mode 3 . This is a passive mode from the patient's point of view, and the robot position controller was implemented with a conventional PD joint compensator.

Notice that the coefficient $k$ is empirically set to 1.0 in order to hardly impose the constraint. The subject cannot deviate from the imposed coordination without applying large forces on the exoskeleton.

TABLE II

SUMMARY OF THE DIFFERENT MODES

\begin{tabular}{|c|c|c|c|c|c|}
\hline Mode & Robot & Gravity comp. & Position cntrl & Therapist & C $(k)$ \\
\hline 1 & - & - & - & - & $\mathbf{0}$ \\
\hline 2 & $\checkmark$ & $\checkmark$ & - & - & $\mathbf{0}$ \\
\hline 3 & $\checkmark$ & $\checkmark$ & - & $\checkmark$ & $\mathbf{0}$ \\
\hline 4 & $\checkmark$ & $\checkmark$ & - & - & $\mathbf{C}_{n}(1.0)$ \\
\hline 5 & $\checkmark$ & $\checkmark$ & - & - & $\mathbf{C}_{t}(1.0)$ \\
\hline 6 & $\checkmark$ & $\checkmark$ & $\checkmark$ & - & $\mathbf{0}$ \\
\hline
\end{tabular}

Table II presents a summary of the modes of control and interactions applied during the complete protocol. Note that the friction compensation was not used because it induced additional inertial phenomena on the exoskeleton affecting its movement. This did not affect the transparency [56]. An example is presented in Fig. 5 with data from Patient $\$ 5$ pointing to target 2 for four modes. These four pictures demonstrate clear differences in the arm posture while the hand position is similar. On the upper-right picture, the therapist constrains the motion in order to reduce shoulder abduction (the elbow remains lower) while on the lower-right picture, the robot programmed to reproduce the therapist instructions performs similarly (lower elbow as compared to the left column pictures).

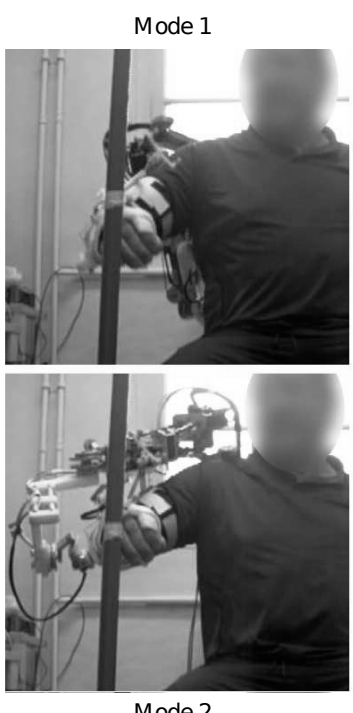

Mode 2

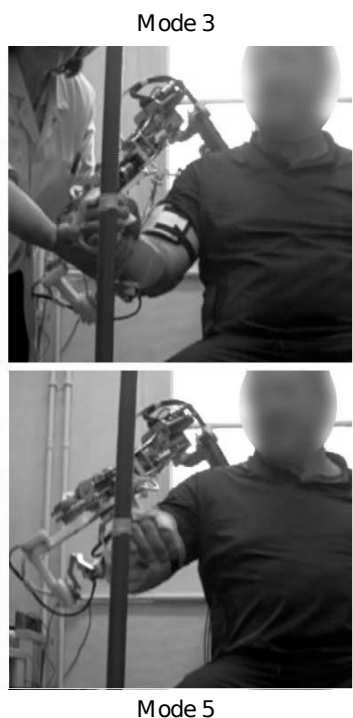

Fig. 5. Patient $\sharp 5$ pointing to target 2 under several control modes. Mode 1 : w/o robot; Mode 2: w/ robot programmed to be transparent ; Mode 3: w/ the therapist imposing the movement while the patient is connected to the robot in the transparent control mode ; Mode 5: w/ robot applying the therapeutic constraint.

\section{E. Data analysis}

The movement was recorded using a Polaris Tracking Optical System (NDI, Ontario) fixed on a beam $1.4 \mathrm{~m}$ above the exoskeleton. Two rigid clusters of 4 reflective markers were fixed on each splint and another one on the beam just above the patient's shoulder. This last cluster provided a reference frame (X rightward, $\mathrm{Y}$ forward and $\mathrm{Z}$ upward) for the measure of the displacements of the Polaris clusters at $60 \mathrm{~Hz}$. Appropriate geometrical computations allowed calculation of the positions of the center of the splint fixed on the patient's arm (UA) and the extremity of the splint fixed on the patient's forearm which corresponds approximately to the endpoint (EP) for pointing (Fig. 3).

Further, analyses were performed in order to quantify the effect of the mode of control on the final values of the exoskeleton joints as well as the impact on the upper limb at the time of pointing. To that purpose, an interactive graphical display was used to examine the time course of the robot data $\left(\mathbf{q}=\left[\mathbf{q}_{1} \cdots \mathbf{q}_{4}\right]^{T}\right.$ and $\left.\dot{\mathbf{q}}=\left[\dot{\mathbf{q}}_{1} \cdots \dot{\mathbf{q}}_{4}\right]^{T}\right)$, the trajectory of the upper arm (UA) and the end point of the forearm (EP) calculated from the Polaris system data. The time of onset of the reaching movement was visually determined for each of the three repetitions, as well as the end of the reach when the patient touched the target or as close as possible (reach end: $\mathrm{RE})$. We then quantified the position $\mathbf{q}$ of each DoF of the robot and 3D position of the arm (UA) and of the endpoint (EP) at RE. The duration of the reach was also calculated.

These dependent variables were analysed using a three factor analysis of variance (ANOVA) with the factors "Mode", "Target" and "Repetition" as independent variables. Statistical analyses were performed on the data of the 6 patients who could make active voluntary movements. 


\section{RESUlts}

\section{A. Inter-joint coordination in hemiparetic patients}

We first studied the applicability of using PCA on joint velocity vectors to define synergies for hemiparetic patients. Fig. 6, 1st column, shows the percentage of the first three PCs for mode 2 (active), averaged for the 6 patients who performed all tasks.

The three first PCs explained, on average, around $96.4 \%$ of the observed joint velocities. These results were similar to those observed in healthy subjects [25] and confirm the existence of joint coupling (synergies) when pointing tasks are performed by hemiparetic patients. Interestingly, when the patient's arms were moved passively by the physical therapist, a coupling relationship between joint velocities was also observed (Fig. 6, mode 3, 4th column, 97.3\% of motion explained by the three first PCs).

TABLE III

$\psi$ AND RATIO VALUES FOR EACH SUBJECT.

\begin{tabular}{|c||c||c|}
\hline Subject & $\psi\left(\mathbf{C}_{n}, \mathbf{C}_{t}\right)$ & $r$ \\
\hline \hline 1 & 0.93 & $7.1 \%$ \\
3 & 0.25 & $11.8 \%$ \\
4 & 1.00 & $3.2 \%$ \\
5 & 0.71 & $14.1 \%$ \\
6 & 0.59 & $1.9 \%$ \\
7 & 0.51 & $12.9 \%$ \\
\hline Mean & 0.66 & $8.5 \%$ \\
\hline
\end{tabular}

Furthermore, in the second column of Table III, the values of the measure $\psi$ between the natural constraint and the therapist constraint for the 6 patients is represented. It can be seen that large inter individual differences occur, reflecting the large variability of the impairments between the 6 patients as well as the adaptation of the movement proposed by the therapist to a given patient.

\section{B. Respect of the applied constraints}

The control law (13) was used to impose different contraints on the patients in modes 4 and 5. These constraints are respectively defined as the natural constraint $\mathbf{C}_{n}$ (calculated from the active movements in mode 2) and the therapeutic constraint $\mathbf{C}_{t}$ defined by movements imposed by the therapist in mode 3 .

It is interesting to observe that in all the modes using the robot assistance, movements consisted of only 3 principal components (Fig. 6, 2nd and 5th column), similarly to previous observations in healthy subjects [25].

In all the modes, the first 3 PCs represent at least $96 \%$ of the movement variance for the 4 different targets. Moreover, this percentage was higher in modes 4 and 5 (constrained modes) than in mode 2 (measuring mode). This shows that the constraints applied in modes 4 and 5 (even the therapeutic constraint which was not natural for the patient), appeared to be well respected with percentages higher than $99.5 \%$ and with small standard deviation values. Values of $r$ ratio (Eq. 8) for the 6 subjects who carried out the whole protocol are presented in the third column of Table III. In this case, $r$ is

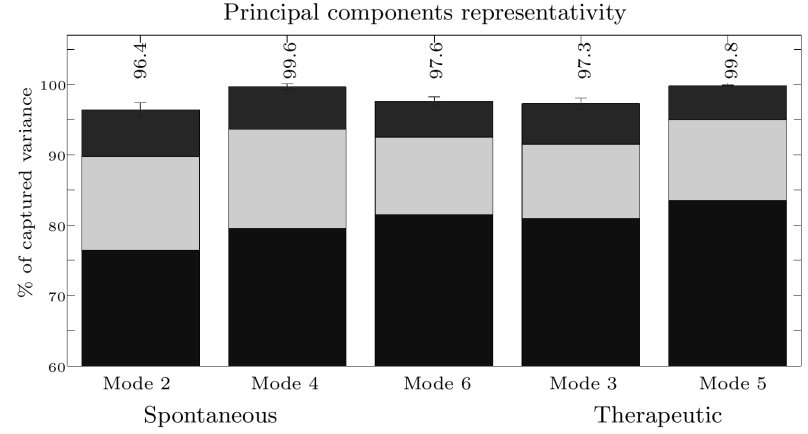

Fig. 6. Percentage of representation of each of the first 3 PCs, and sum for the first 3, for each mode, averaged for the 6 selected patients. Mode 2: w/ robot in transparent mode (no correction applied); Mode 4: w/ robot applying the natural patient constraint; Mode $6 \mathrm{w} /$ robot playing the therapeutic trajectory in position control mode; Mode 3: w/ robot with therapist guiding the movement; Mode 5: w/ robot applying the therapeutic constraint .

given by:

$$
r=\frac{\psi\left(\mathbf{C}_{5}^{T}, \mathbf{C}_{t}^{T}\right)}{\psi\left(\mathbf{C}_{n}^{T}, \mathbf{C}_{t}^{T}\right)}
$$

with $\mathbf{C}_{5}$ the constraint matrix built from PCA of data obtained during mode 5. The values of $r$ shown in Table III indicate that the therapeutic constraints were well respected by all 6 subjects. They are lower than $15 \%$ (with a mean of $8.5 \%$ ) which means that all the patients carried out the different movements with a coordination at least 7 times closer to the therapeutic contraints than to the natural constraints.

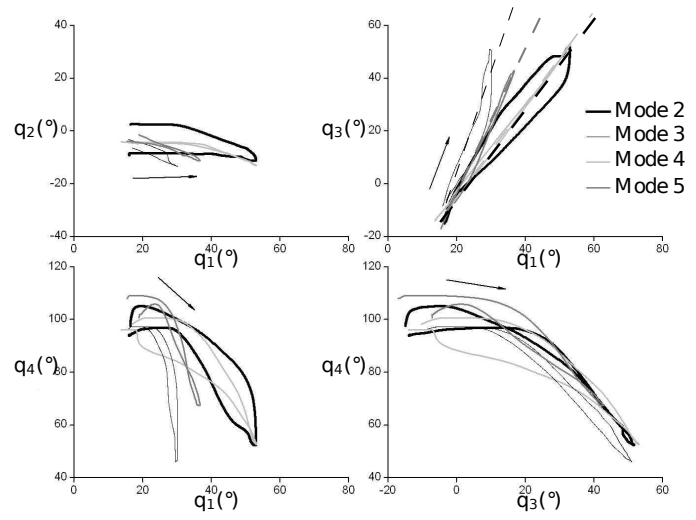

Fig. 7. Time co-variation of the robot DoF during one reach and return movement in the different modes (upper part: $\mathbf{q}_{2}$ and $\mathbf{q}_{3}$ as a function of $\mathbf{q}_{1}$; lower part: $\mathbf{q}_{4}$ as a function of $\mathbf{q}_{1}$ and $\mathbf{q}_{3}$ ). The arrows indicate the direction of reach and doted straight lines indicate the direction of the co-variation.

Joint angle coordination is shown in Fig. 7. It demonstrates that $\mathbf{C}_{n}$ (light grey lines) constrains the trajectory relative to the transparent mode (thick black lines) but not by reference to the direction of the co-variation in the DoF. In contrast, the therapist's guidance (thin black lines) as well as $\mathbf{C}_{t}$ (middle grey lines) both modify the direction of the co-variation of the DoF. The effect of $\mathbf{C}_{t}$ is intermediary between that of the spontaneous coordination and the therapist's guidance. 
C. Consequences of the control law on the kinematics of pointing movements

Statistical analyses were performed on the data from the 6 patients who could make active voluntary movements.

1) Two factor ANOVA (with mode and target as factors) comparing the control condition without the robot (mode 1) with the transparent mode (mode 2) showed that the 3D position of the arm was altered by the robot indicating that it was not fully transparent [53]. The positions varied with the target $(p<0.0001$; except for the forward position of the endpoint EPy $p<0.05)$. Reaching with the robot limited the antero-posterior position of the arm at the time of pointing (UAy $p<0.05$ ), exaggerated its lateral position (UAx $p<0.0001$ ); but did not modify its height nor the final position of the endpoint.

2) The three factor ANOVA performed on robot and kinematic data showed that there were no differences between repetitions, whatever the condition (patient moving actively or not), the mode or the target. Thus, the repetition factor was not considered for further analysis.

3) A two factor ANOVA (with mode and target as factors) was performed for the passive modes. We compared the data obtained when the patient's arm motion was imposed by the therapist (mode 3) or by the robot (mode 6). Fig. 8 (Left) shows that, as expected, the final configuration of the four joints varied significantly with the target $(p<0.0001)$. In contrast, there were no differences between modes, except for $\mathbf{q}_{2}(p<0.01)$ which was greater when it was imposed by the robot than by the therapist (respectively $-17^{\circ} \pm 1.5$ and $\left.-11^{\circ} \pm 1.3\right)$. The robot did not move the upper limb as high as the therapist: on average $\mathrm{UAz}=62.5 \mathrm{~mm}$ for the $\operatorname{arm}(p<$ $0.0001)$ and $\mathrm{EPz}=67.0 \mathrm{~mm}$ for the forearm $(p<0.01)$. There were no interactions. The duration of the reaching was shorter when the movement was produced by the robot than with a therapist, but this result may be simply due to the arbitrary choice of the replay velocity.

4) The experiments with imposed motions were also applied without particular difficulty to the patient $\sharp 2$ who had complete paralysis. The analysis of robot data showed that the control law could satisfactorily reproduce the action of the therapist (the final values of $\mathbf{q}_{1}, \mathbf{q}_{2}, \mathbf{q}_{3}$ were slightly but not significantly smaller and $\mathbf{q}_{2}$ slightly greater).

5) Similarly, a two factor ANOVA (with mode and target as factors) was performed on the modes when the patient moved actively (modes 2, 4 and 5). Figure 8 (Right) confirms that the final configuration varied significantly with the target $(p<0.0001)$. There were significant differences between modes only for $\mathbf{q}_{1}(p<0.0001)$ and no interaction between the effects of mode and target. Post Hoc Tukey-Kramer test showed that the final configuration of $\mathbf{q}_{1}$ at the time of pointing recorded with the "therapeutic constraint" $\left(\mathbf{C}_{t}\right)$ (mode 5 ) differed from the data recorded at mode 2 (mean difference $10.4^{\circ}$ ) and at mode 4 (with the natural constraint $\mathbf{C}_{n}$, mean difference $8.7^{\circ}$ ). The analysis of the $3 \mathrm{D}$ position of the arm and forearm pointed out to the expected differences due to the target $(p<0.0001)$ but not to a significant effect of mode, nor

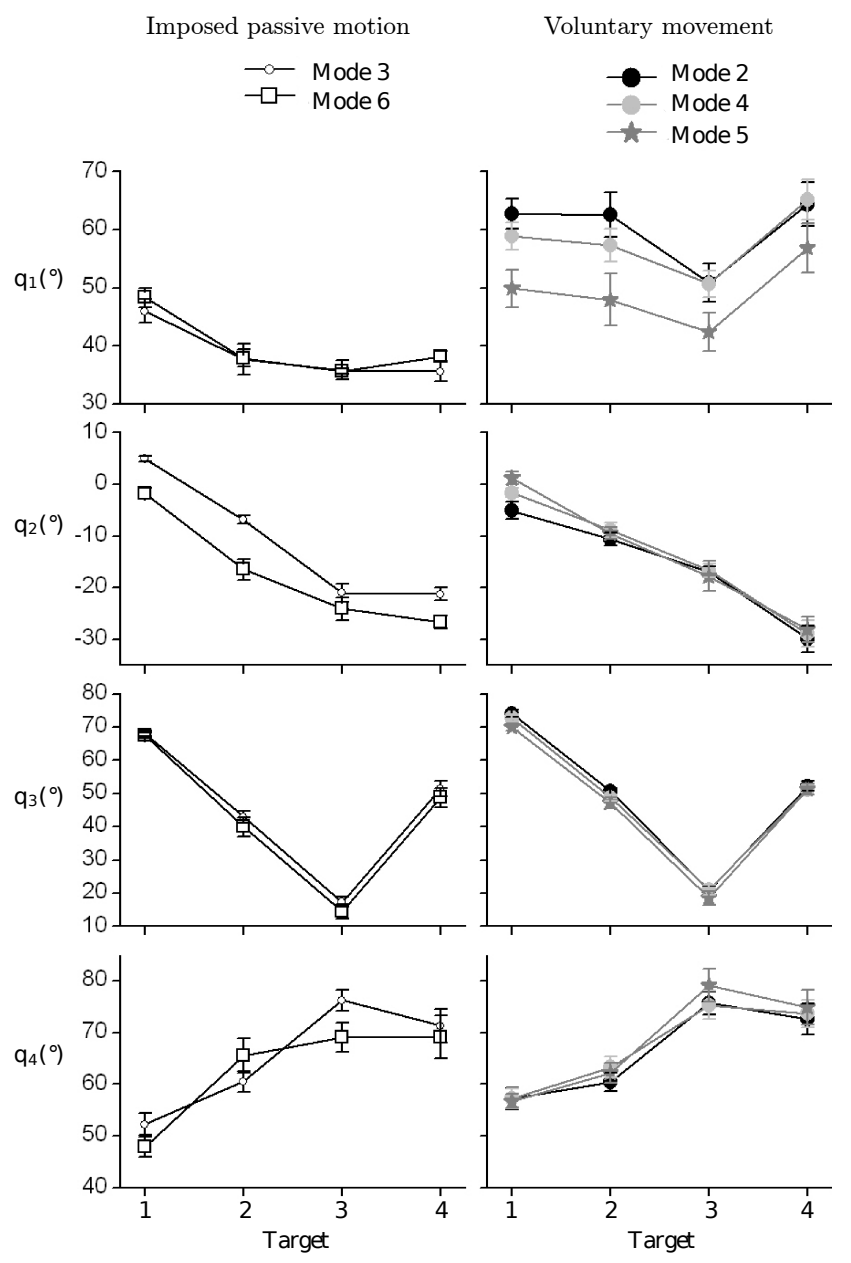

Fig. 8. Final posture of the robot, measured in the different modes (separated into 2 categories, passive and active), for each target averaged for the 6 subjects. Each graph represents one of the robot DoF.

interactions between modes and targets. The duration of the reach varied significantly with the target (from $1.7 \mathrm{~s}$ for Target 2 to 2.1 s for Target $1, p<0.001$ ) but did not vary significantly with the mode; there were no interactions.

6) The final robot configurations were compared in three conditions : the passive arm motion (grouping data of modes 3 and 6) active movement (grouping data of modes 2 and 4) and "therapeutic constraints" $\left(\mathbf{C}_{t}\right)$ (mode 5) (two factor ANOVA with condition and target as factors). There were significant differences in $\mathbf{q}_{1}(p<0.0001)$ between voluntary movements (mean of all targets: $59^{\circ} \pm 1.9$ ) and imposed movements $\left(39.7^{\circ} \pm 1.4\right)$; the effect of the "therapeutic constraint" being intermediary $\left(49.2^{\circ} \pm 3.4\right)$ and significantly different from the two others. There was no significant effect of condition for $\mathbf{q}_{2}-\mathbf{q}_{4}$. To summarize, the above analyses demonstrate that the new viscous constraint (mode 5) did not significantly affect the endpoint trajectory compared with the other active modes $(2$ and 4) but significantly changes q1. The values of q1 obtained with mode 5 are closer to those desired, based on the therapist movement (mode3).

7) The difference in $\mathbf{q}_{1}$ between the robot in transparent (mode 2) and therapeutic mode (mode 5) varied with the 
patient. It was significantly correlated with the coefficient $r$ calculated in part V-B for Target 2-4 but not for Target 1 . The coefficients of correlation varied with the target : 0.57 for Target $1,0.927$ for Target $2(p<0.01), 0.828$ for Target 3 $(p=0.05)$ and 0.825 for Target $4(p=0.05)$.

8) An informal debriefing of the session showed that the exercises with the robotic device were well accepted and found to be interesting by the patients. No particular problems were encountered during the procedure, even with the most affected patient.

\section{DISCUSSION}

Rehabilitation consisting of active-assisted mobilization of the upper limb by the therapist was mainly developed in the classical Bobath approach. This approach insists on the production of "good quality" coordination and on the prevention of abnormal contractions, through precise interactions between the patient and therapist using specific handling techniques. The PCA analysis (Sec. V-A) shows that the therapist imposed movements using a consistent coordination pattern. This confirms the previous study by Culmer et al. [24] in which passive movements by a therapist were recorded using the IPAM robot. They observed regular common trends in the trajectory of the limbs and in the torque imposed on the upper limb joints. In complement, we quantify here the synergies imposed by the therapist and we demonstrate that they are substantially different to the spontaneous coordination of the patient.

A second observation is that the control law can impose similar constraints to the therapist, improving the patients' movement. In particular, the "therapeutic constraint" may push the coordination of the movement in the direction desired by the therapist as recorded during mode 3 .

The other results provided in this paper describe the effects of these constraints on the final configuration of the robot and upper limb at the end of the reach. In brief, the effect of the "therapeutic constraint" resulted in a significant decrease of the final angle of $\mathbf{q}_{1}$, without significantly modifying the trajectory of the endpoint. This result corresponds well with the typical therapeutic aim of reducing excessive elbow elevation which was therefore also one of the aims of the robotic controller, as illustrated in Fig. 5. Although the effect was not directly measured on the human arm it is likely that the present command is able to correct, at least partially, the excessive arm elevation performed by hemiparetic patients during reaching.

Most likely those modifications are due to an equilibrium between the configuration planned by the patients and the modification of the viscous field interacting with their upper limb during the execution of the movement [57]. The absence of modification during the three repetitions rules out an immediate learning effect or "one trial adaptation" effect [58]. The present experiment did not include enough movement repetition nor post-effect measures to analyze a possible learning process.

In addition, it is interesting to observe differences in $\mathbf{q}$ and in $\mathrm{EPz}$ when the robot was moved by the therapist (mode 3) and when the robot reproduced the movement autonomously (mode 6). These differences may be due to technical reasons (e.g. the moderated stiffness of the position controller, which was intentionally chosen for safety reasons or dynamic effects due to the velocity of the replay). Moreover mode 3 was performed in an interactive situation which was not the case for mode 6 which may lead to a different behavior of the robot-patient system.

\section{CONCLUSION AND PERSPECTIVES}

In this article, we presented a novel robot control approach, which takes advantage of the exoskeleton structure in order to apply force fields at the joint level. This method integrates an explicit model of inter-joint coordination based on a linear relationship between joint velocities. This tool allows the characterisation of both active patient motions and passive, therapist-assisted motions. Experiments conducted with hemiparetic patients showed that, the robot was able to modify spontaneous coordination patterns in individual hemiparetic patients, without modifying end-effector kinematics.

Exoskeleton-mediated therapy using viscous fields combines the classical Bobath therapy and the motor relearning approach. On the one hand, this concept allows finely tuned corrections of coordination during voluntary movements as recommended by Bobath therapists. In addition, it provides a better knowledge of the sensorimotor mechanisms operating in this empirical method [24], [50]. On the other hand, the future developments will capitalize on the acquired knowledge on robot assisted motor learning. Robot therapy may allow a much larger repetition of movements in a larger variety of sensorimotor contexts. This step corresponds to a generalization of the knowledge acquired during robot assisted learning at the end-point level to the question of joint coordination and redundancy.

Along these lines, it is noteworthy to mention that because the present approach is based on copying the therapist's action, it avoids explicit computation of a reference upper limb configuration, a scientific question that is as yet unresolved. Further multidisciplinary experiments in healthy subjects and hemiparetic patients are needed to specify the therapeutic indications of this device and modes of control for rehabilitative purposes.

\section{REFERENCES}

[1] R. Launois, M. Giroud, A.C. Megnigbeto, K. Le Lay, G. Presente, M.H Mahagne, and et al. Estimating the cost-effectiveness of stroke units in France compared with conventional care. Stroke, 35(3):770-775, 2004.

[2] H. Nakayama, H.S. Jorgensen, H.O. Raaschou, and T. S. Olsen. Recovery of upper extremity function in stroke patients: the Copenhagen Stroke Study. Arch Phys Med Rehabil, 75(4):394-398, 1994.

[3] G. Kwakkel, B. Kollen, and E. Lindeman. Understanding the pattern of functional recovery after stroke: facts and theories. Restor Neurol Neurosci, 22:281-299, 2004.

[4] Y.S. Lam, D.W. Man, S.F. Tam, and P.L. Weiss. Virtual reality training for stroke rehabilitation. NeuroRehabilitation, 21(3):245-253, 2006.

[5] M. Popovic and D. Popovic. Cloning biological synergies improves control of elbow neuroprostheses. Engineering in Medicine and Biology Magazine, IEEE, 20(1):74-81, 2001.

[6] D.J. Reinkensmeyer, J.L. Emken, and Cramer S.C. Robotics, motor learning, and neurologic recovery. Annu Rev Biomed Eng., 6:497-525, 2004.

[7] G. Kwakkel, B.J. Kollen, and H.I. Krebs. Effects of Robot-Assisted therapy on upper limb recovery after stroke: A systematic review. Neurorehabil Neural Repair, 22(2):111-121, April 2008. 
[8] S.E. Fasoli, H.I. Krebs, and N. Hogan. Robotic technology and stroke rehabilitation: translating research into practice. Top Stroke Rehabil, 11(4):11-19, 2004.

[9] R. Riener, M. Frey, M. Bernhardt, T. Nef, and G. Colombo. Humancentered rehabilitation robotics. In Proceedings of the 9th International Conference on Rehabilitation and Robotics, pages 319-22, 2005.

[10] M. Schoone, P. van Os, and A. Campagne. Robot-mediated Active Rehabilitation (ACRE) A user trial. In IEEE 10th International Conference on Rehabilitation Robotics, 2007. ICORR 2007, pages 477-481, 2007.

[11] S.E. Fasoli, H.I. Krebs, J. Stein, W.R. Frontera, and N. Hogan. Effects of robotic therapy on motor impairment and recovery in chronic stroke. Archives of physical medicine and rehabilitation, 84(4):477-482, 2003.

[12] B.T. Volpe, P.T. Huerta, J.L. Zipse, A. Rykman, D. Edwards, L. Dipietro, N. Hogan, and H.I. Krebs. Robotic devices as therapeutic and diagnostic tools for stroke recovery. Archives of neurology, 66(9):1086, 2009.

[13] B.T. Volpe, D. Lynch, A. Rykman-Berland, M. Ferraro, M. Galgano, N. Hogan, and H.I. Krebs. Intensive sensorimotor arm training mediated by therapist or robot improves hemiparesis in patients with chronic stroke. Neurorehabil Neural Repair, 22:305-310, 2008.

[14] A.C. Lo, P.D. Guarino, L.G. Richards, J.K. Haselkorn, G.F. Wittenberg, D.G. Federman, R.J. Ringer, T.H. Wagner, H.I. Krebs, B.T. Volpe, et al. Robot-assisted therapy for long-term upper-limb impairment after stroke. New England Journal of Medicine, 362(19):1772-1783, 2010.

[15] MC Cirstea, AB Mitnitski, AG Feldman, and MF Levin. Interjoint coordination dynamics during reaching in stroke. Experimental Brain Research, 151(3):289-300, 2003.

[16] L. Dipietro, HI Krebs, SE Fasoli, BT Volpe, J. Stein, C. Bever, and N. Hogan. Changing motor synergies in chronic stroke. Journal of Neurophysiology, 98(2):757-768, 2007.

[17] R.A.R.C. Gopura, K. Kiguchi, and Yang Li. SUEFUL-7 : a 7DOF upper-limb exoskeleton robot with muscle-model-oriented EMG-based control. In Intelligent Robots and Systems, 2009. IROS 2009. IEEE/RSJ International Conference on, pages 1126-1131, 2009.

[18] EA Brackbill, Y. Mao, SK Agrawal, M. Annapragada, and V.N. Dubey. Dynamics and Control of a 4-dof Wearable Cable-driven Upper Arm Exoskeleton. In Robotics and Automation, 2009. ICRA 2009. IEEE International Conference on, 2009.

[19] M. Laffranchi, N.G. Tsagarakis, F. Cannella, and Caldwell D.G. Antagonistic and series elastic actuators : a comparative analysis on the energy consumption. In In Intelligent Robots and Systems, 2009. IROS 2009. IEEE/RSJ International Conference on, pages 5678-5684, 2009.

[20] T. Nef and R. Riener. ARMin-design of a novel arm rehabilitation robot. In Rehabilitation Robotics, 2005. ICORR 2005. 9th International Conference on, pages 57-60, 2005.

[21] P. Garrec, JP Friconneau, Y. Measson, and Y. Perrot. ABLE, an innovative transparent exoskeleton for the upper-limb. In IEEE/RSJ International Conference on Intelligent Robots and Systems, 2008. IROS 2008, pages 1483-1488, 2008.

[22] M. Mihelj, T. Nef, and R. Riener. A novel paradigm for patientcooperative control of upper-limb rehabilitation robots. Advanced Robotics, 21(8):843-867, 2007.

[23] C.R. Carignan, M.P. Naylor, and S.N. Roderick. Controlling shoulder impedance in a rehabilitation arm exoskeleton. In IEEE International Conference on Robotics and Automation Pasadena, 2008.

[24] P.R. Culmer, A.E. Jackson, S.G. Makower, J.A. Cozens, M.C. Levesley, M. Mon-Williams, and B. Bhakta. A novel robotic system for quantifying arm kinematics and kinetics: Description and evaluation in therapistassisted passive arm movements post-stroke. Journal of Neuroscience Methods, 197(2):259-269, April 2011.

[25] V. Crocher, N. Jarrasse, A. Sahbani, A. Roby-Brami, and G. Morel. Changing human upper-limb synergies with an exoskeleton using viscous fields. In IEEE International Conference on Robotics and Automation (ICRA'11), pages 4657-4663, Shanghai, China, 2011

[26] C.J. Mottram, N.L. Suresh, C.J. Heckman, M.A. Gorassini, and W.Z Rymer. Origins of abnormal excitability in biceps brachii motoneurons of spastic-paretic stroke survivors. Journal of Neurophysiology, 102(4):2026-2038, 2009.

[27] S. Brunnstrom. Movement Therapy in Hemiplegia: A Neurophysiological Approach. New York: Harper \& Row, 1970.

[28] L. Dipietro, H.I. Krebs, S.E. Fasoli, B.T. Volpe, and N. Hogan. Submovement changes characterize generalization of motor recovery after stroke. Cortex, 45(3):318 -324, 2009.

[29] R.F. Beer, J.P. Dewald, and W.Z. Rymer. Deficits in the coordination of multijoint arm movements in patients with hemiparesis: evidence for disturbed control of limb dynamics. Experimental brain research, 131(3):305-319, 2000.
[30] M.F. Levin. Interjoint coordination during pointing movements is disrupted in spastic hemiparesis. Brain, 119:281-293, 1996

[31] S. Micera, J. Carpaneto, F. Posteraro, L. Cenciotti, M. Popovic, and P. Dario. Characterization of upper arm synergies during reaching tasks in able-bodied and hemiparetic subjects. Clinical Biomechanics, 20(9):939-946, 2005.

[32] J. Dewald and R.F. Beer. Abnormal joint torque patterns in the paretic upper limb of subjects with hemiparesis. Muscle \& nerve, 24(2):273283, 2001.

[33] A. Roby-Brami, A. Feydy, M. Combeaud, E.V. Biryukova, B. Bussel, and M.F. Levin. Motor compensation and recovery for reaching in stroke patients. Acta Neurol Scand, 107:369-381, 2003.

[34] R.F. Beer, M.D. Ellis, B.G. Holubar, and J.P.A. Dewald. Impact of gravity loading on post-stroke reaching and its relationship to weakness. Muscle \& nerve, 36(2):242, 2007.

[35] N.K. Musampa, P.A. Mathieu, and M.F. Levin. Relationship between stretch reflex thresholds and voluntary arm muscle activation in patients with spasticity. Experimental brain research, 181(4):579-597, 2007.

[36] R.D. Trumbower, V.J. Ravichandran, M.A. Krutky, and E.J. Perreault. Contributions of altered stretch reflex coordination to arm impairments following stroke. Journal of Neurophysiology, 104(6):3612-3624, 2010.

[37] N. Bernstein. The co-ordination and regulation of movements. Pergamon New York, 1967.

[38] M. Latash. Synergy. Oxford University Press, 2008.

[39] T. Bockemuhl, N.F. Troje, and V. Durr. Inter-joint coupling and joint angle synergies of human catching movements. Human Movement Science, 29(1):73-93, 2010.

[40] J.P. Scholz and G. Schner. The uncontrolled manifold concept: Identifying control variables for a functional task. Experimental brain research, 126(3):289-306, 1999.

[41] D. Domkin, J. Laczko, M. Djupsjobacka, S. Jaric, and M.L. Latash Joint angle variability in 3D bimanual pointing: Uncontrolled manifold analysis. Experimental brain research, 163(1):44-57, 2005.

[42] D.S. Reisman and J.P. Scholz. Aspects of joint coordination are preserved during pointing in persons with post-stroke hemiparesis. Brain, 126(11):2510-2527, 2003.

[43] D.S. Reisman and J.P. Scholz. Workspace location influences joint coordination during reaching in post-stroke hemiparesis. Experimental brain research, 170(2):265-276, 2006.

[44] V.S. Huang and J.W. Krakauer. Robotic neurorehabilitation: a computational motor learning perspective. Journal of NeuroEngineering and Rehabilitation, 6:5, 2009.

[45] B. Bobath. Adult hemiplegia: evaluation and treatment. ButterworthHeinemann Medical, 1990.

[46] P.M. van Vliet, N.B. Lincoln, and E. Robinson. Comparison of the content of two physiotherapy approaches for stroke. Clinical rehabilitation, 15(4):398, 2001

[47] PM Van Vliet, NB Lincoln, and A. Foxall. Comparison of bobath based and movement science based treatment for stroke: a randomised controlled trial. Journal of Neurology, Neurosurgery \& Psychiatry, 76(4):503, 2005.

[48] B. Langhammer and J.K. Stanghelle. Can physiotherapy after stroke based on the bobath concept result in improved quality of movement compared to the motor relearning programme. Physiotherapy Research International, 16(2):69-80, 2011.

[49] M.F. Levin and E. Panturin. Sensorimotor integration for functional recovery and the bobath approach. Motor Control, 15:285-301, 2011.

[50] M.F. Levin, S.M. Michaelsen, C.M. Cirstea, and A. Roby-Brami. Use of the trunk for reaching targets placed within and beyond the reach in adult hemiparesis. Experimental brain research, 143(2):171-180, 2002.

[51] S.M. Michaelsen, A. Luta, A. Roby-Brami, and M.F. Levin. Effect of trunk restraint on the recovery of reaching movements in hemiparetic patients. Stroke, 32(8):1875-1883, 2001.

[52] D.B. Popovic, M.B. Popovic, T. Sinkjær, A. Stefanovic, and L. Schwirtlich. Therapy of paretic arm in hemiplegic subjects augmented with a neural prosthesis: a cross-over study. Canadian journal of physiology and pharmacology, 82(8-9):749-756, 2004.

[53] N. Jarrassé, M. Tagliabue, J.V.G. Robertson, A. Maiza, V. Crocher, A. Roby-Brami, and G. Morel. A methodology to quantify alterations in human upper limb movement during co-manipulation with an exoskeleton. Neural Systems and Rehabilitation Engineering, IEEE Transactions on, 18(4):389-397, 2010.

[54] V. Crocher, A. Sahbani, and G. Morel. Imposing joint kinematic constraints with an upper limb exoskeleton without constraining the hand motion. In IEEE/RSJ International Conference on Intelligent Robots and Systems (IROS'10), pages 5028-5033, Taipei, Taiwan, 2010. 
[55] N. Jarrassé and G. Morel. On the kinematic design of exoskeletons and their fixations with a human member. In Proceedings of Robotics: Science and Systems. Citeseer, 2010.

[56] N. Jarrasse, M. Tagliabue, J. Robertson, A. Maiza, V. Crocher, A. RobyBrami, and G. Morel. A methodology to quantify alterations in human upper limb movement during co-manipulation with an exoskeleton. IEEE in Transactions on Neural Systems \& Rehabilitation Engineering, 18(4), 2010. to appear.

[57] N. St-Onge and A.G. Feldman. Referent configuration of the body: a global factor in the control of multiple skeletal muscles. Experimental brain research, 155(3):291-300, 2004.

[58] D.L. Weeks, MP Aubert, A.G. Feldman, and M.F. Levin. One-trial adaptation of movement to changes in load. Journal of neurophysiology, 75(1):60, 1996.

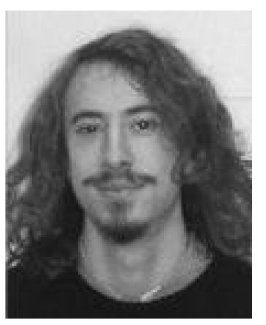

Vincent Crocher received the M.S. degree in electronics and computer science, in 2008 , and is currently working toward the Ph.D. degree at the University Pierre et Marie Curie since October 2008. $\mathrm{He}$ is working on exoskeleton control for upper limb rehabilitation at the Institute of Intelligent Systems and Robotics (ISIR).

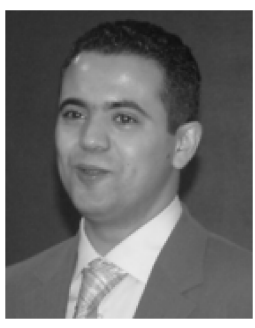

Anis Sahbani is an Associate Professor at Pierre et Marie Curie University (Paris 6). He holds his research activities at the Intelligent Systems and RoboticsInstitute (ISIR-CNRS-UMR 7222). He has been the Principal Investigator for several research projects, and has been exploring different topics on robotics control,computer vision and, in particular, manipulation planning. He obtained an Electrical Engineering Degree from Ecole Nationale dIngnieurs de Tunis, in 1998, and MS in Automation and Computer Engineering, in 1999. He received his $\mathrm{PhD}$ in Robotics and Computer Science from the University Paul Sabatier, LAAS-CNRS, in 2003.

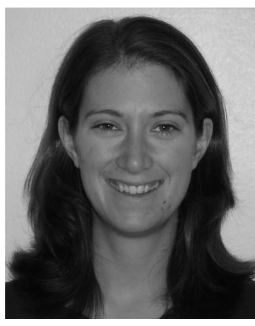

Johanna Robertson received a BSc (Hons) in Physiotherapy in 1999 at Robert Gordon University, Aberdeen, Scotland. After specialising in neurological rehabilitation, she undertook an MSc in Motor Control followed by a $\mathrm{PhD}$ on the subject of alterations in upper limb motion following hemiparesis. She is currently working as a Research Therapist in Raymond Poincar Hospital, Garches, France. Her particular interest is in understanding motor deficits of the upper limb following neurological diseases for the development of more effective rehabilitation techniques, including technological devices.

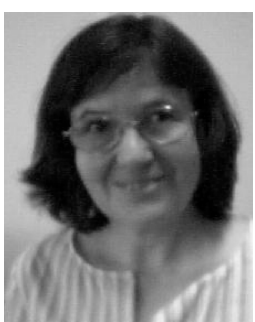

Agnès Roby-Brami received the M.D. degree from the University ParisDescartes, in 1982, and the M.Sc. and the Ph.D. degrees and University Habilitation in Neurosciences from Pierre et Marie Curie University, Paris, France, in 1982, 1991, and 2001, respectively. She is a physician who performed her residency in Paris Hospitals during 19771982. She was appointed as a Research Associate at INSERM in 1983 and promoted to Research Director in 2005. Her rst research interest was in the eld of clinical neurophysiology after which she focused on upper limb motor control, disability, and recovery in cerebral and spinal injured patients. She is the author or coauthor of 83 peer-reviewed academic publications, and 44 contributions to edited books. She is also actively involved in several Academic Networks concerning research on disability.

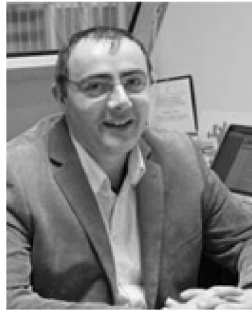

Guillaume Morel received the M.S. degree in electrical engineering and the Ph.D. degree in mechanical engineering from the University Pierre et Marie Curie, Paris, France, in 1990 and 1994, respectively. After a postdoc at the Massachusetts Institute of Technology and a rst assistant professorship in Strabsourg, he came back to Paris in 2001. Over these years, his research interests have been force feedback control and visual servoing of robots, with, for the last decade, a particular focus on medical applications. He now heads a multlidisciplinary group developing devices aimed at assisting gesture through the concept of comanipulation. 